\section{IDDF2021-ABS-0167 REAL-WORLD EFFICACY AND SAFETY OF SOFOSBUVIR/VELPATASVIR/VOXILAPREVIR IN NS5A-INHIBITOR EXPERIENCED PATIENTS: AN INTERNATIONAL MULTICENTER STUDY FROM ASIA}

\begin{abstract}
${ }^{1}$ Yu Jun Wong ${ }^{*},{ }^{2}$ Rajneesh Kumar, ${ }^{3}$ Chen Hua Liu, ${ }^{3}$ Jia Horng Kao, ${ }^{4}$ Vicky Wing-Ki Hui,
\end{abstract} ${ }^{4}$ Grace Wong, ${ }^{5}$ Prem Harichander Thurairajah. ${ }^{1}$ Changi General Hospital, Singapore; DukeNUS Medicine Academic Clinical Program, Singhealth, Singapore; ${ }^{2}$ Singapore General Hospital, Singapore; ${ }^{3}$ National Taiwan University Hospital, Taiwan; ${ }^{4}$ Chinese University of Hong Kong, Hong Kong; ${ }^{5}$ National University of Singapore, Singapore

\subsection{6/gutjnl-2021-IDDF.99}

Background Sofosbuvir/Velpatasvir/Voxilaprevir (SOF/VEL/VOX) was approved as salvage therapy for NS5A-experienced hepatitis $\mathrm{C}$ virus $(\mathrm{HCV})$ infected patients. However, the realworld data of SOF/VEL/VOX remained limited in Asia. We aim to analyse the real-world efficacy and safety of SOF/ VEL/VOX among NS5A-experienced Asian HCV patients.

Methods This was a cross-sectional, multicenter, international study assessing the efficacy of retreatment using SOF/VEL/ VOX among Asian HCV patients with prior direct-acting antiviral (DAA) failure. Our primary aim is sustained-virological response 12 weeks after completion of treatment (SVR12). Data on safety and treatment outcomes were also recorded.

Results Nineteen patients were included from 5 hospitals. Median age was 57, 84\% were male, 47\% were ex-IVDU and $63 \%$ had liver cirrhosis. Among 12 patients with liver cirrhosis, $75 \%$ were Child-Turcotte-Pugh Class A and 25\% were Class B. Commonest genotype (GT) requiring retreatment using SOF/VEL/VOX was GT3 (68\%), followed by GT1, GT2 and GT6 (11\%). Prior DAA include SOF/VEL (79\%), HARVONI (11\%), Glecaprevir/Pibrentasvir (5\%) and Daclatasvir/ Asunaprevir (5\%).

The overall SVR12 rate by intention-to-treat and per-protocol (PP) analysis was $84.1 \%$ and $94.1 \%$, respectively. Two patients were demised before treatment completion from HCC progression and septic shock, and were excluded from PP analysis. The SVR12 was not significantly different in patients with GT3 (GT3: 91\%, non-GT3: 100\%, p=0.647). The only patient with virological failure had compensated GT3 cirrhosis with portal hypertension after completed 12 weeks of SOF/ VEL/VOX. Significant improvement observed in median serum ALT, AST and bilirubin following SVR12 $(\mathrm{p}<0.05)$

Conclusions In this real-world international study, we found that SOF/VEL/VOX is an efficacious and safe salvage regimen among NS5A-experienced HCV patients in Asia.

\section{IDDF2021-ABS-0172 EFFICACY AND SAFETY OF DIRECT ORAL ANTICOAGULANTS VERSUS VITAMIN $K$ ANTAGONISTS FOR PORTAL VEIN THROMBOSIS IN CIRRHOSIS: A SYSTEMATIC REVIEW AND META-ANALYSIS}

${ }^{1}$ Sherilyn Zi Hui Liew* ${ }^{2}$ Jin Hean Koh, ${ }^{1}$ Gin Kee Ng, ${ }^{2}$ Hui Ting Liu, ${ }^{3}$ Yew Chong ${ }^{4}$ Andrea De Gottardi, ${ }^{1} Y u$ Jun Wong. ${ }^{1}$ Department of Gastroenterology and Hepatology, Changi General Hospital, Singapore; ${ }^{2}$ Yong Loo Lin School of Medicine, National University of Singapore, Singapore; ${ }^{3}$ Education Resource Centre, Medical Board, Singapore General Hospital, Singapore; ${ }^{4}$ Gastroenterology and Hepatology, Ente Ospedaliero Cantonale, Lugano, Switzerland

10.1136/gutjpl-2021-IDDF. 100

Background Portal vein thrombosis (PVT) is associated with a higher risk of liver decompensation, variceal bleeding and long-term mortality in cirrhosis patients. Vitamin $\mathrm{K}$ antagonists (VKAs) have a narrow therapeutic range and require frequent dose titration, and the bleeding risk of VKAs in cirrhosis patients is not accurately reflected in the international normalized ratio (INR). Recent American Association for the Study of Liver Diseases (AASLD) guidelines recommend direct-acting oral anticoagulants (DOACs) in cirrhosis patients with nontumoral PVT. However, the comparative efficacy between DOACs and VKAs for the treatment of cirrhosis with nontumoral PVT currently remains unknown. We aim to perform a systematic review and meta-analysis to compare the efficacy and safety of DOACs versus VKAs to treat cirrhosis patients with non-tumoral PVT.

Methods We performed a systematic search of six electronic databases and selected all studies comparing DOACs with VKAs in the treatment of PVT in cirrhosis patients. The primary outcome was either complete or partial PVT recanalization. Secondary outcomes were PVT progression, major bleeding, variceal bleeding and death. (IDDF2021-ABS-0172 Figure 1)

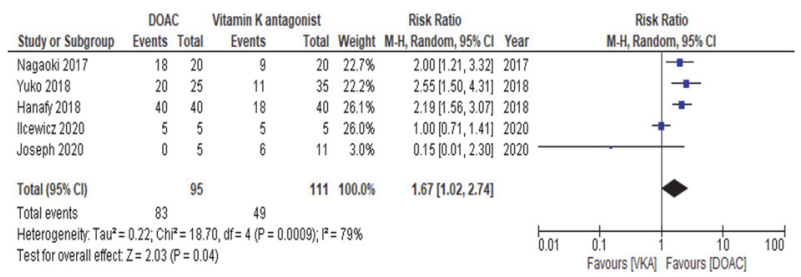

Abstract IDDF2021-ABS-0172 Figure 1 Pooled rate of portal vein thrombosis recanalization (partial or complete)

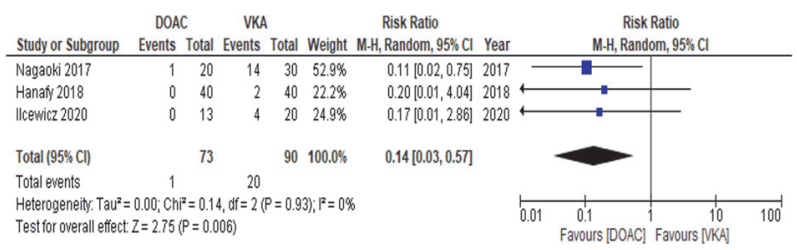

Abstract IDDF2021-ABS-0172 Figure 2 Pooled risk of portal vein thrombosis progression

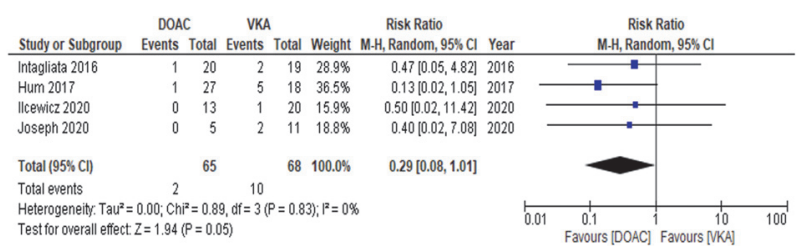

Abstract IDDF2021-ABS-0172 Figure 3 Pooled risk of major bleeding

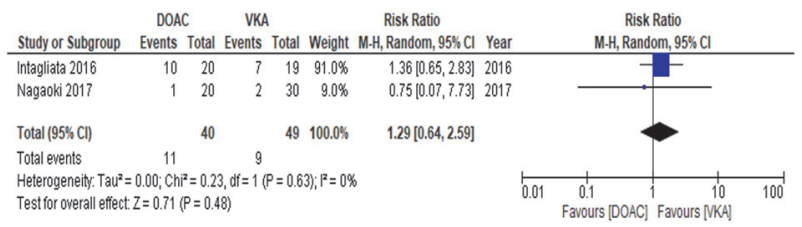

Abstract IDDF2021-ABS-0172 Figure 4 Pooled risk of variceal bleeding 